Supporting Information

for

\title{
Insecticide Risk in United States Surface Waters: Drivers and Spatiotemporal Modeling
}

\author{
Jakob Wolfram ${ }^{\dagger}$, Sebastian Stehle ${ }^{\dagger, \ddagger}$, Sascha Bub ${ }^{\dagger}$, Lara L. Petschick ${ }^{\dagger}$, Ralf Schulz ${ }^{*}$ \\ †iES Landau, Institute for Environmental Sciences, University of Koblenz-Landau, \\ Fortstrasse 7, D-76829 Landau, Germany \\ *Eusserthal Ecosystem Research Station, University of Koblenz-Landau, Birkenthalstrasse \\ 13, D-76857 Eusserthal, Germany \\ *Phone: +49634128031327; e-mail: schulz@uni-landau.de
}

Pages S1 - S1 1

Tables S1 - S7

Figures S1 - S3 


\section{Methods:}

\section{List of degradation products}

In the following, a list of all degradation products that were deemed to be potentially relevant to aquatic environments, is provided and more detailed descriptions for their inclusion in this meta-analysis can be found in Wolfram et al. ${ }^{1}$ :

Endosulfan sulfate, azinphos-methyl oxygen analog, 3,5,6-trichloro-2-pyridinol, chlorpyrifos oxygen analog, chlorpyrifos oxon, diazoxon, diazinon oxon, malaoxon, isomalathion, malathion dicarboxylic acid, malathion monocarboxylic acid, paraoxon, methyl paraoxon, 3hydroxycarbofuran, 3-ketocarbofuran, carbofuran phenol, alpha-R-deltamethrin, 6chloronicotinic acid, imidacloprid guanidine, imidacloprid olefin, imidacloprid olefinicguanidine, imidacloprid urea, o-p-dichlorobenzophenone, $\mathrm{p}$-p-dichlorobenzophenone, 1,1bis(p-chlorophenyl-)2,2-dichloroethanol, dichlorobenzhydrol, hydroxyldichlorobenzophenone, aldicarb sulfoxide, aldoxycarb, aldicarb sulfone, N-(2-chloro-5thizolylmethyl)-N'-nitroguanidine, 1-methyl-2-nitro-3-(tetrahydro-3-furylmethyl)-guanidine, 1-methylguanidinium chloride, 3-(methylamino)-9-oxa-2-aza-4-azoniabicyclone-3enehydrogen succinate, 1-methyl-2-nitroguanidine, 1-methyl-3-(tetrahydro-3furylmethyl)guanidine, 1-methyl-3-(tetrahydro-3-furylmethyl)-guanidinium dihydrogen phosphate, fipronil sulfone, fipronil sulfide, and desulfinyl fipronil.

\section{Multiple linear regression validation}

MLRs were validated following suggestions of Unwin ${ }^{2}$ and references therein. Briefly, the normality distribution of the residuals was checked visually using histograms and QQ-Plots, while the independence of errors was tested using the Durbin-Watson-Test with $1>d>3$ representing thresholds that would suggest autocorrelation. ${ }^{3}$ Homoscedasticity was assessed visually by plotting standardized residuals versus their fitted values. Additionally, the 
significance of all regression coefficients was checked using White's heteroscedasticity corrected covariance matrix ${ }^{4}$ and by calculating regression coefficient $95 \%$ confidence intervals. Both tests did not reveal any departure from the initial MLR results. Outliers were assessed with studentized residuals and standardized residuals, while potential leverage points were assessed using hat-values, DFFit, Cook's distance and covariance ratios. Multicolinearity was assessed using variance inflation factors with a threshold level of 3 . For MLRs using factorial attributes with more than one degree of freedom (df), generalized variance inflation factors were calculated (GVIF, see Fox and Monette ${ }^{5}$ ). GVIFs were then transformed back via squaring, allowing comparison with traditionally used threshold levels (i.e. VIF < 3). Only substances with more than 20 MICs were used for all MLRs, and no violations of concern became apparent.

\section{Internal validation via simulation}

Following aforementioned standard validation exercises, every model was further tested for robustness and validity applying internal validation techniques, ${ }^{6,7}$ using a simulation approach (i.e. bootstrapping, $n=10,000)$ of randomly drawn data (with replacements).

\section{Surface water risk (individual substances)}

Detailed summary statistics about bootstrapping simulation runs $(n=10,000)$ of the linear model for individually measured MICs in freshwaters $\left(n=1,833, \mathrm{R}^{2}\right.$ adj. $\left.=62.17, \mathrm{p}<0.001\right)$ are provided in Table S3. Bootstrapping simulation runs $(n=10,000)$ and their resulting adjusted $R^{2}$ are summarized in Figure $S 3$.

\section{Sediment risk (individual substances)}

Detailed summary statistics about bootstrapping simulation runs $(n=10,000)$ of the linear model for individually measured MICs in sediments $\left(n=478, \mathrm{R}^{2}\right.$ adj. $\left.=48.10, \mathrm{p}<0.001\right)$ are 
provided in Table S6. Bootstrapping simulation runs $(n=10,000)$ and their resulting adjusted $\mathrm{R}^{2}$ are summarized in Figure $\mathrm{S} 2$.

Surface water risk (maxTU)

Detailed summary statistics about bootstrapping simulation runs $(n=10,000)$ of the maximum toxicant pressure (i.e. maxTU) model for surface waters $\left(n=496, \mathrm{R}^{2}\right.$ adj. $=75.17$, $\mathrm{p}<0.001)$ are provided in Table S7. Bootstrapping simulation runs $(n=10,000)$ and their resulting adjusted $\mathrm{R}^{2}$ are summarized in Figure $\mathrm{S} 3$.

\section{References}

1. Wolfram, J.; Stehle, S.; Bub, S.; Petschick, L. L.; Schulz, R., Meta-Analysis of Insecticides in United States Surface Waters: Status and Future Implications. Environ. Sci. Technol. 2018, 52, (24), 14452-14460.

2. Unwin, A., Discovering Statistics Using R by Andy Field, Jeremy Miles, Zoë Field. International Statistical Review 2013, 81, (1), 169-170.

3. Durbin, J.; Watson, G. S., Testing for serial correlation in least squares regression. II. Biometrika 1951, 38, (1/2), 159-177.

4. White, H., A Heteroskedasticity-Consistent Covariance Matrix Estimator and a Direct Test for Heteroskedasticity. Econometrica 1980, 48, (4), 817-838.

5. Fox, J.; Monette, G., Generalized collinearity diagnostics. Journal of the American Statistical Association 1992, 87, (417), 178-183.

6. Harrell Jr, F. E., Regression modeling strategies: with applications to linear models, logistic and ordinal regression, and survival analysis. Springer: 2015.

7. $\quad$ Steyerberg, E. W.; Harrell Jr, F. E.; Borsboom, G. J.; Eijkemans, M.; Vergouwe, Y.; Habbema, J. D. F., Internal validation of predictive models: efficiency of some procedures for logistic regression analysis. J. Clin. Epidemiol. 2001, 54, (8), 774-781.

8. $\quad$ Lewis, K. A.; Tzilivakis, J.; Warner, D. J.; Green, A., An international database for pesticide risk assessments and management. Human and Ecological Risk Assessment: An International Journal 2016, 22, (4), 1050-1064. 


\section{List of Tables}

Table S1: Regulatory threshold levels (RTL) for freshwater $\left(\mathrm{RTL}_{\mathrm{fw}}\right)$, sediments $\left(\mathrm{RTL}_{\mathrm{sed}}\right)$ and estuaries $\left(\mathrm{RTL}_{\mathrm{est}}\right)$ for all 32 parent compounds. Only insecticide degradates with reported concentrations are listed and provided with a RTL. A full list of degradates of potential environmental concern can be found in the first paragraph of the SI. Please note, this table can also be found in Wolfram et al. ${ }^{1}$

\begin{tabular}{|c|c|c|c|c|}
\hline Insecticide class & Insecticide & $\begin{array}{l}\mathrm{RTL}_{\mathrm{fw}} \\
(\mu \mathrm{g} / \mathrm{L})\end{array}$ & $\begin{array}{l}\mathrm{RTL}_{\mathrm{sed}} \\
(\mu \mathrm{g} / \mathrm{kg})\end{array}$ & $\begin{array}{l}\mathrm{RTL}_{\text {est }} \\
(\mu \mathrm{g} / \mathrm{L})\end{array}$ \\
\hline \multirow[t]{2}{*}{ Organochlorine } & Dicofol & 26.5 & 3680 & 7.55 \\
\hline & Endosulfan & 0.05 & 3.84 & 0.02 \\
\hline \multirow[t]{6}{*}{ Organophosphate } & Azinphos-methyl & 0.08 & 0.89 & 0.105 \\
\hline & Chlorpyrifos & 0.03 & 16 & 0.0175 \\
\hline & Diazinon & 0.105 & 76 & 2.1 \\
\hline & Malathion & 0.295 & 2.56 & 1.1 \\
\hline & Parathion-ethyl & 0.02 & 0.325 & 0.0535 \\
\hline & Parathion-methyl & 0.485 & 20.8 & 0.175 \\
\hline \multirow[t]{3}{*}{ Carbamate } & Aldicarb & 10 & & 6 \\
\hline & Carbaryl & 0.85 & 4.4 & 2.85 \\
\hline & Carbofuran & 1.115 & 1.72 & 2.3 \\
\hline \multirow[t]{15}{*}{ Pyrethroid } & Bifenthrin & 0.0002465 & 0.25 & 0.001985 \\
\hline & Cyfluthrin & 0.0125 & 0.53 & 0.0012 \\
\hline & Cypermethrin & 0.0018 & 0.308 & 0.002375 \\
\hline & $\zeta$-cypermethrin & 0.0018 & 0.308 & 0.002375 \\
\hline & Deltamethrin & 0.0001 & 0.48 & 0.00185 \\
\hline & Esfenvalerate & 0.000424 & 7.4 & 0.00233 \\
\hline & Fenpropathrin & 0.001525 & 12.4 & 0.0105 \\
\hline & Fenvalerate & 0.016 & 2.2 & 0.004 \\
\hline & $\lambda$-cyhalothrin & 0.00015 & 0.31 & 0.00245 \\
\hline & Permethrin & 0.0033 & 7.4 & 0.009 \\
\hline & Resmethrin & 0.14 & & 0.115 \\
\hline & $\tau$-fluvalinate & 0.155 & & 0.003 \\
\hline & Tefluthrin & 0.03 & 11.6 & 0.0265 \\
\hline & Tetramethrin & 1.85 & & 1.85 \\
\hline & Tralomethrin & 0.0195 & & 0.4225 \\
\hline \multirow[t]{5}{*}{ Neonicotinoid } & Acetamiprid & 10.5 & & 33 \\
\hline & Clothianidin & 11 & & 26.5 \\
\hline & Dinotefuran & 49550 & & 395 \\
\hline & Imidacloprid & 0.385 & & 16.5 \\
\hline & Thiamethoxam & 17.5 & 10 & 3450 \\
\hline Phenylpyrazole & Fipronil & 0.11 & 16 & 0.07 \\
\hline \multirow[t]{4}{*}{ Degradate } & Endosulfan sulfate & 0.05 & 73 & 0.02 \\
\hline & Aldicarb sulfoxide & 21.5 & & \\
\hline & Aldicarb sulfone & 184.5 & & \\
\hline & Fipronil sulfone & 0.36 & 9.1 & 0.28 \\
\hline
\end{tabular}




$\begin{array}{lccc}\text { Fipronil sulfide } & 1.065 & 29 & 0.0385 \\ \text { Desulfinyl fipronil } & 100 & 200 & 0.75\end{array}$

Table S2: List of covariates and attributes with their respective data type and availability in percent that were extracted from publications and assigned to individual MICs.

\begin{tabular}{|c|c|c|c|}
\hline Covariate & Data type & Description & Available \% \\
\hline Substance name & factor & Name of detected insecticide & 100 \\
\hline Chemical class & factor & Insecticide class (e.g. Organophosphate) & 100 \\
\hline TU identifier & integer & Shared identifier per sample & 100 \\
\hline Reference & factor & Name and date of the respective publication & 100 \\
\hline Compartment & integer & $\begin{array}{l}\text { Integer detailing if MIC was detected in the water } \\
\text { phase (1) or sediment phase (2) }\end{array}$ & 100 \\
\hline Sampling interval & numeric & $\begin{array}{l}\text { Time in days between individual samples per } \\
\text { location }\end{array}$ & 77.4 \\
\hline Catchment size & numeric & $\begin{array}{l}\text { Size in } \mathrm{km}^{2} \text { of contributing catchment area per } \\
\text { sampling location }\end{array}$ & 81.7 \\
\hline Sampling date & numeric & Date of sampling event & 98.1 \\
\hline Location name & factor & Name of sampling location & 100 \\
\hline Agricultural produce & factor & List of crops grown in respective catchment & 71.2 \\
\hline Sampling location (state) & factor & $\begin{array}{l}\text { Federal state in which the sampling location is } \\
\text { situated }\end{array}$ & 97.8 \\
\hline Smapling location (county) & factor & County in which the sampling location is situated & 78.7 \\
\hline Water body type & factor & $\begin{array}{l}\text { Water body type (e.g. creek, estuary, river) as } \\
\text { detailed in the publication }\end{array}$ & 95.6 \\
\hline Freshwater & integer & $\begin{array}{l}\text { Integer detailing if the respective water body } \\
\text { contains freshwater (1) or estuarine water (2) }\end{array}$ & 100 \\
\hline Flow type & integer & $\begin{array}{l}\text { Interger detailing if respective water body is flowing } \\
\text { (1) or standing (2) }\end{array}$ & 97 \\
\hline Number of detects & integer & $\begin{array}{l}\text { Number of detections per substance as detailed in the } \\
\text { publication }\end{array}$ & 97.7 \\
\hline Numbe rof non-detects & integer & $\begin{array}{l}\text { Number of non-detections per substance as detailed } \\
\text { in the publication }\end{array}$ & 83.1 \\
\hline Filtration & factor & $\begin{array}{l}\text { Description of filtration procedures used for } \\
\text { pyrethroid samples }\end{array}$ & 95.7 \\
\hline LOD & numeric & Analytical limit of detection for respective substance & 39.9 \\
\hline LOQ & numeric & $\begin{array}{l}\text { Analytical limit of quantification for respective } \\
\text { substance }\end{array}$ & 51.1 \\
\hline Additional pesticides & factor & $\begin{array}{l}\text { Factor indicating if additional pesticides (excluding } \\
\text { insecticides) were analyzed }\end{array}$ & 97.7 \\
\hline Additional insecticides & factor & $\begin{array}{l}\text { Factor indicating if additional insecticides were } \\
\text { analyzed }\end{array}$ & 100 \\
\hline Total pesticides & integer & $\begin{array}{l}\text { Number of total detected pesticides (excluding } \\
\text { insecticides) per sample }\end{array}$ & 100 \\
\hline Total insecticides & integer & Number of total detected insecticides per sample & 65.2 \\
\hline Total Pesticides/Insecticides & factor & $\begin{array}{l}\text { Factor detailing how many pesticides/insecticides } \\
\text { were detected }\end{array}$ & 99 \\
\hline Detected pesticides & factor & List of susbtance names detected per sample & 61.7 \\
\hline Agricultural origin & factor & $\begin{array}{l}\text { Factor describing certainty that MICs originated } \\
\text { from agricultural NPS as detailed in the publication }\end{array}$ & 100 \\
\hline
\end{tabular}




\begin{tabular}{lllr} 
Agricultural proximity & factor & $\begin{array}{l}\text { Factor describing if any information was provided } \\
\text { regarding the proximity of agricultural fields to the } \\
\text { sampling location }\end{array}$ & 60.9 \\
Highest spatial accuracy & integer & $\begin{array}{l}\text { Integer detailing if MIC was attributable to a location } \\
(1), \text { county (2), or the federal state (3) }\end{array}$ & 100 \\
Latitude & numeric & Latitude of sampling location (GPS) & 75.3 \\
Longitude & numeric & Longitude of sampling location (GPS) & 75.3 \\
RTL & numeric & respective regulatory threshold level & 100 \\
RTL class & integer & Integer detailing if MIC was attributable to a \\
& & freshwaters (1), sediments (2), or estuarine waters (3) & 100 \\
\hline
\end{tabular}

Table S3: Detailed summary statistics of model validation simulation runs (bootstrapping, $n=10,000$ ) and resulting bootstrapped $95 \%$ confidence intervals for the freshwater linear regression of individual MIC to RTL ratios. Standardized betas with their respective rank (SBR) and variance inflation factors (VIF) of the main model are provided for reference.

\begin{tabular}{|c|c|c|c|c|c|c|c|}
\hline Predictor variable & $\begin{array}{c}\text { Lower } \\
95 \%- \\
\text { confidence } \\
\text { limit } \\
\end{array}$ & $\begin{array}{c}2^{\text {nd }} \\
\text { quartile } \\
\text { (median) }\end{array}$ & $\begin{array}{c}\text { Upper } \\
95 \%- \\
\text { confidence } \\
\text { limit } \\
\end{array}$ & Sign. ${ }^{1}$ & $\begin{array}{l}\text { Std. } \\
\text { beta }\end{array}$ & $\mathrm{SBR}^{2}$ & VIF \\
\hline Intercept & -2.302 & -1.928 & -1.542 & * & & & \\
\hline Catchment size $\left[\mathrm{km}^{2}\right]^{3}$ & -0.183 & -0.151 & -0.120 & * & -0.146 & 4 & 1.897 \\
\hline Sampling interval $[\mathrm{d}]^{3}$ & -0.227 & -0.199 & -0.170 & * & -0.189 & 3 & 1.311 \\
\hline Time [y] & -0.064 & -0.057 & -0.050 & * & -0.287 & 2 & 2.283 \\
\hline toxicity-normalized use $\left[\mathrm{kg} \times \mathrm{RTL}^{-1}\right]^{3}$ & 0.350 & 0.385 & 0.422 & * & 0.381 & 1 & 1.987 \\
\hline Burst factor & 0.011 & 0.015 & 0.019 & $*$ & 0.131 & 5 & 2.310 \\
\hline Irrigated agricultural land-use [\%] & 0.289 & 0.427 & 0.560 & * & 0.101 & 6 & 1.448 \\
\hline Organophosphates & -0.034 & 0.242 & 0.518 & & & & 1.653 \\
\hline Pyrethroids & 0.944 & 1.231 & 1.516 & $*$ & & & 1.653 \\
\hline Neonicotinoids & 0.130 & 0.436 & 0.726 & * & & & 1.653 \\
\hline Phenylpyrazole & 0.807 & 1.235 & 1.678 & $*$ & & & 1.653 \\
\hline
\end{tabular}

Table S4: Comparison of insecticides' stability in days (i.e. $\mathrm{DT}_{50}$ ) in different compartments and the resulting delta in days. Data was obtained from Lewis et al. ${ }^{8}$.

\begin{tabular}{lrrr}
\multicolumn{1}{c}{ Substance } & $\begin{array}{c}\mathrm{DT}_{50} \text { in days } \\
\text { (sediment) }\end{array}$ & $\begin{array}{c}\mathrm{DT}_{50} \text { in days } \\
\text { (water) }\end{array}$ & Delta \\
\hline Malathion & 0.4 & 0.4 & 0 \\
Diazinon & 10.4 & 4.3 & 6.1 \\
Endosulfan & $N / A$ & $N / A$ & $N / A$ \\
Chlorpyrifos & 36.5 & 5 & 31.5 \\
Bifenthrin & 161 & 8 & 153 \\
K-cyhalothrin & 15.1 & 0.24 & 14.86
\end{tabular}




\begin{tabular}{lrrr} 
Esfenvalerate & 56 & 30 & 26 \\
Fenpropathrin & 28 & 1 & 27 \\
Carbaryl & 5.8 & 3.1 & 2.7 \\
Carbofuran & 9.7 & 6.1 & 3.6 \\
Permethrin & 40 & 23 & 17 \\
Deltamethrin & 65 & 17 & 48 \\
Cypermethrin & 17 & 3 & 14 \\
Fenvalerate & $N / A$ & $N / A$ & $N / A$ \\
Parathion-methyl & 4.3 & 3.5 & 0.8 \\
Cyfluthrin & 8 & 1 & 7 \\
Cypermethrin-zeta & 2 & 0.1 & 1.9 \\
Fipronil & 68 & 54 & 14 \\
Desulfinyl-Fipronil & $N / A$ & $N / A$ & $N / A$ \\
Fipronil sulfide & $N / A$ & $N / A$ & $N / A$ \\
Fipronil sulfone & $N / A$ & $N / A$ & $N / A$ \\
Dicofol & 29 & $N A$ & $N / A$ \\
\hline
\end{tabular}

Table S5: Toxic modes of action for respective insecticide classes used in the present study.

\begin{tabular}{ll} 
Insecticide class & Toxic Mode of Action (TMoA) \\
\hline Organochlorine & GABA-gated chloride channel antagonist \\
Organophosphate & Acetylcholinesterase inhibitor \\
Carbamate & Acetylcholinesterase inhibitor \\
Pyrethroid & Sodium channel modulators \\
Neonicotinoid & Nicotinic acetylcholine receptor agonists \\
Phenylpyrazole & GABA $_{\mathrm{A}}$-gated chloride channel antagonist \\
\hline
\end{tabular}

Table S6: Detailed summary statistics of model validation simulation runs (bootstrapping, $n=10,000$ ) and resulting bootstrapped $95 \%$ confidence intervals for the sediment linear regression of individual MIC to RTL ratios. Standardized betas with their respective rank (SBR) and variance inflation factors (VIF) of the main model are provided for reference.

\begin{tabular}{|c|c|c|c|c|c|c|c|}
\hline Predictor variable & $\begin{array}{c}\text { Lower } \\
95 \%- \\
\text { confidence } \\
\text { limit }\end{array}$ & $\begin{array}{c}2^{\text {nd }} \\
\text { quartile } \\
\text { (median) }\end{array}$ & $\begin{array}{c}\text { Upper } \\
95 \%- \\
\text { confidence } \\
\text { limit }\end{array}$ & Sign. ${ }^{1}$ & $\begin{array}{l}\text { Std. } \\
\text { beta }\end{array}$ & $\mathrm{SBR}^{2}$ & VIF \\
\hline Intercept & -3.239 & -2.414 & -1.602 & * & & & \\
\hline Catchment size $\left[\mathrm{km}^{2}\right]^{2}$ & -0.142 & -0.101 & -0.061 & $*$ & -0.097 & 6 & 1.150 \\
\hline Sampling interval $[\mathrm{d}]^{2}$ & -0.085 & -0.053 & -0.021 & $*$ & -0.075 & 7 & 1.257 \\
\hline Time $[y]$ & -0.085 & -0.067 & -0.049 & $*$ & -0.215 & 2 & 1.274 \\
\hline Toxicity-normalized use $[\mathrm{kg} \times \mathrm{RTL}-1]^{2}$ & 0.087 & 0.188 & 0.281 & * & 0.170 & 3 & 1.808 \\
\hline Lentic bodies [factor] & -0.528 & -0.367 & -0.210 & * & -0.098 & 5 & 1.336 \\
\hline Irrigated agricultural land-use [\%] & 0.312 & 0.481 & 0.656 & * & 0.126 & 4 & 1.236 \\
\hline $\mathrm{KOC}[\mathrm{L} / \mathrm{kg}]^{2}$ & 0.617 & 0.769 & 0.965 & * & 0.506 & 1 & 2.879 \\
\hline Organophosphates [factor] & -1.326 & -0.783 & -0.301 & * & & & 1.368 \\
\hline
\end{tabular}




\begin{tabular}{lllll} 
Pyrethroids [factor] & -0.946 & -0.421 & 0.063 & 1.368 \\
Phenylpyrazole [factor] & -2.117 & -0.798 & 0.379 & 1.368 \\
\hline
\end{tabular}

${ }^{1}$ Determined based on confidence level overlap

${ }^{2}$ Predictor variable was transformed by the decadal logarithm

${ }^{3}$ Ranked standardized betas of linear model

Table S7: Detailed summary statistics of model validation simulation runs (bootstrapping, $n=10,000$ ) and resulting bootstrapped 95\% confidence intervals for the mixture toxicity (maxTU, freshwaters) linear regression of individual MIC to RTL ratios. Standardized betas with their respective rank (SBR) and variance inflation factors (VIF) of the main model are provided for reference.

\begin{tabular}{|c|c|c|c|c|c|c|c|}
\hline Predictor variable & $\begin{array}{c}\text { Lower } \\
95 \%- \\
\text { confidence } \\
\text { limit }\end{array}$ & $\begin{array}{c}2^{\text {nd }} \\
\text { quartile } \\
\text { (median) }\end{array}$ & $\begin{array}{c}\text { Upper } \\
95 \%- \\
\text { confidence } \\
\text { limit }\end{array}$ & Sign. ${ }^{1}$ & $\begin{array}{l}\text { Std. } \\
\text { beta }\end{array}$ & $\mathrm{SBR}^{2}$ & VIF \\
\hline Intercept & -2.219 & -1.736 & -1.243 & * & & & \\
\hline Catchment size $\left[\mathrm{km}^{2}\right]^{3}$ & -0.393 & -0.314 & -0.238 & * & -0.246 & 3 & 2.071 \\
\hline Sampling interval $[\mathrm{d}]^{3}$ & -0.194 & -0.130 & -0.063 & * & -0.106 & 7 & 1.470 \\
\hline Burst factor & 0.010 & 0.018 & 0.025 & * & 0.138 & 6 & 2.180 \\
\hline Time $[y]$ & -0.074 & -0.063 & -0.053 & * & -0.269 & 2 & 1.180 \\
\hline Toxicity-normalized use $\left[\mathrm{kg} \times \mathrm{RTL}^{-1}\right]^{3}$ & 0.394 & 0.448 & 0.503 & * & 0.411 & 1 & 1.490 \\
\hline Number of detected substances & 0.050 & 0.073 & 0.097 & * & 0.151 & 5 & 1.665 \\
\hline Irrigated agricultural land-use [\%] & 0.726 & 0.989 & 1.254 & * & 0.209 & 4 & 1.453 \\
\hline
\end{tabular}

${ }^{1}$ Determined based on confidence level overlap

${ }^{2}$ Ranked standardized betas of linear model

${ }^{3}$ Predictor variable was transformed by the decadal logarithm 


\section{List of figures}

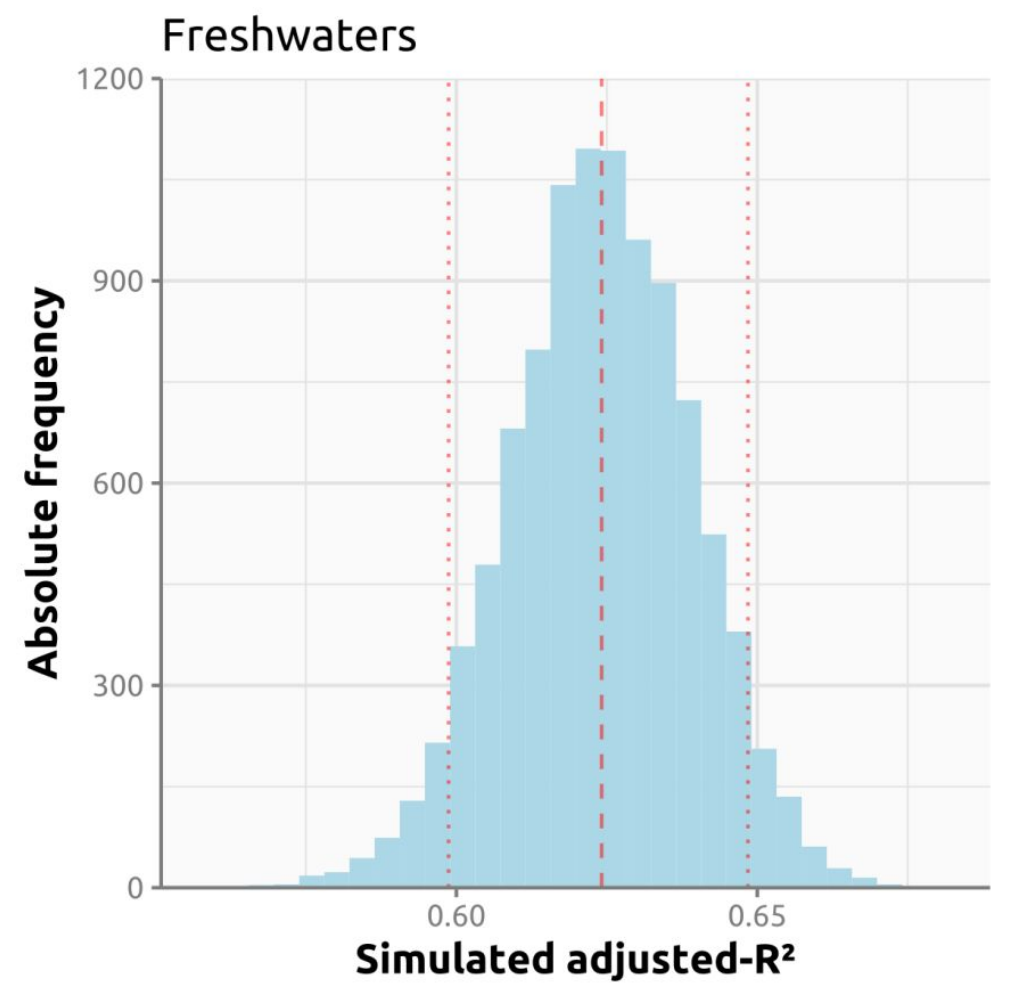

Figure S1: Frequency plot detailing the distribution of bootstrap simulated $(n=10,000)$ adj. $\mathrm{R}^{2}$ of the multiple linear regression for insecticide risks in freshwaters. Bootstrapped upper and lower $95 \%$ confidence intervals are depicted by dotted (orange) lines and the median is depicted by a red dashed line. 


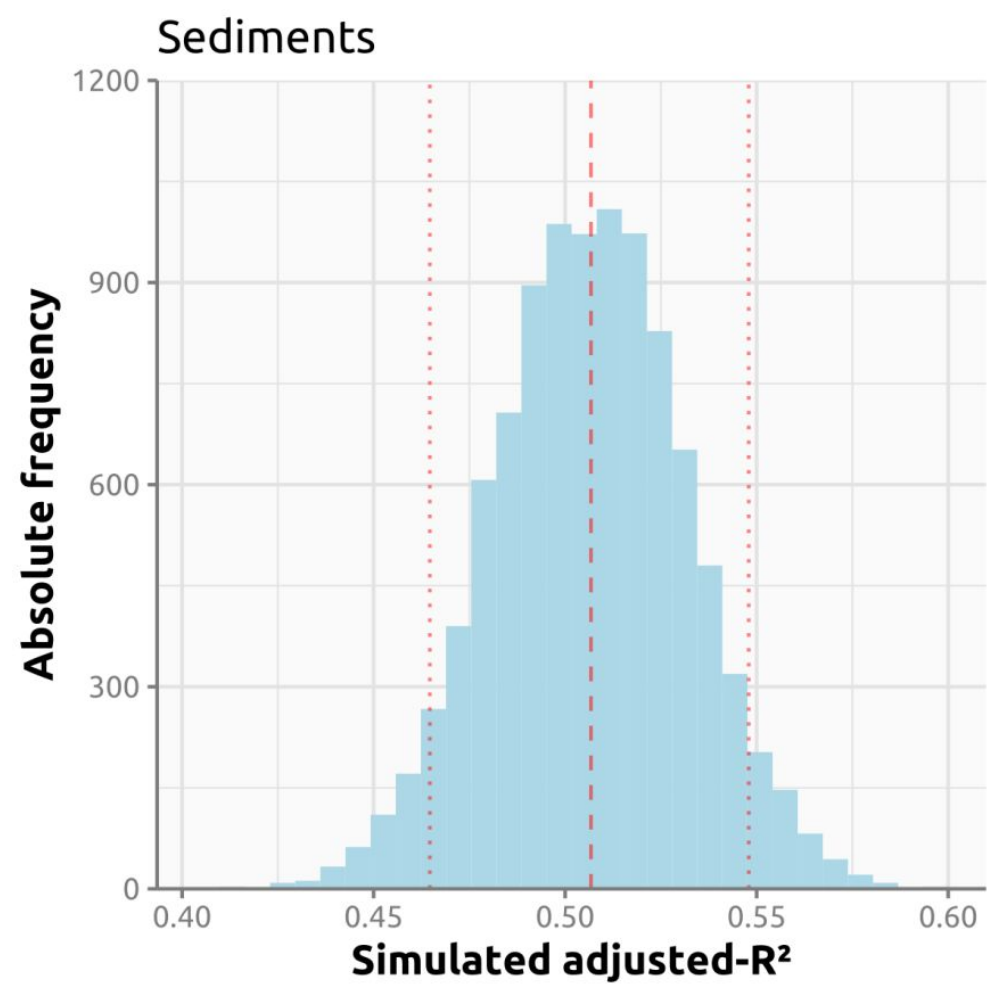

Figure S2: Frequency plot detailing the distribution of bootstrap simulated $(n=10,000)$ adj. $\mathrm{R}^{2}$ of the multiple linear regression for insecticide risks in sediments. Bootstrapped upper and lower $95 \%$ confidence intervals are depicted by dotted (orange) lines and the median is depicted by a red dashed line.

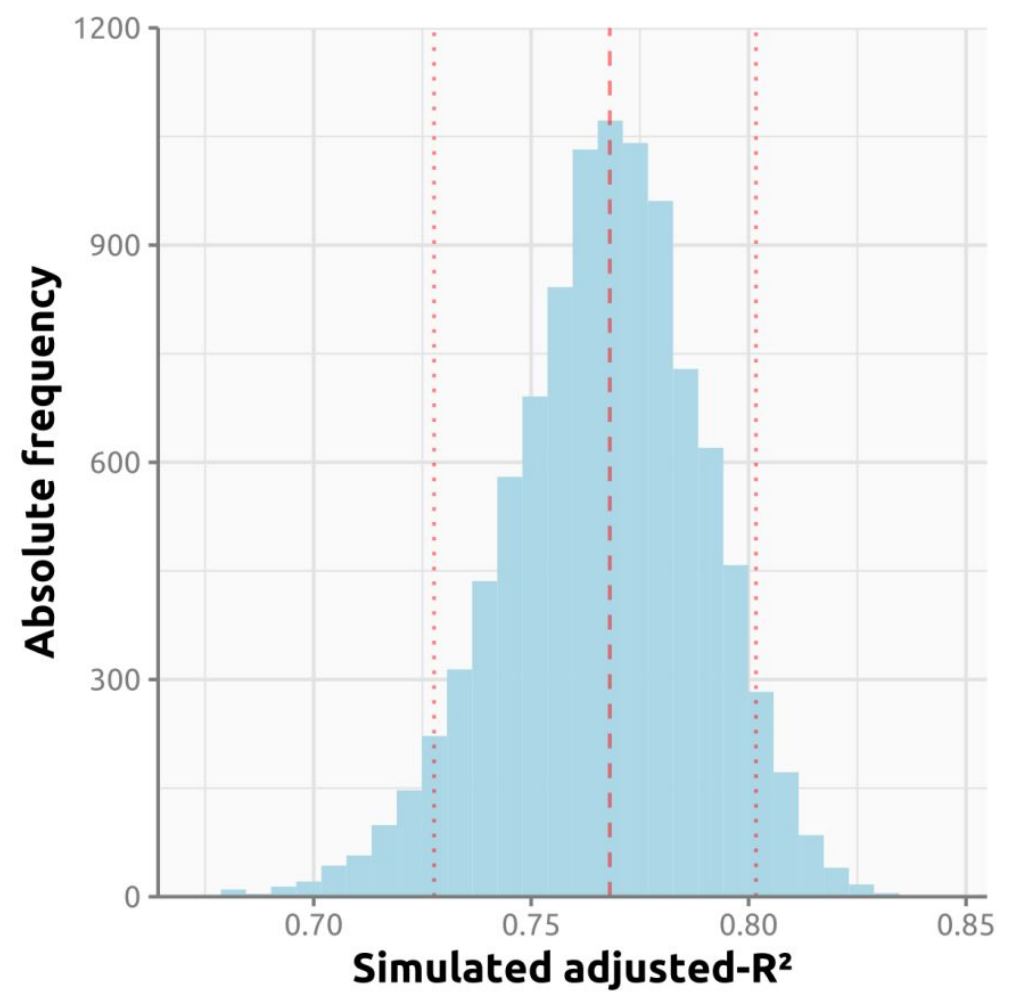

Figure S3: Frequency plot detailing the distribution of bootstrap simulated $(n=10,000)$ adj. $\mathrm{R}^{2}$ of the maxTU multiple linear regression. Bootstrapped upper and lower $95 \%$ confidence intervals are depicted by dotted (orange) lines and the median is depicted by a red dashed line. 